\title{
Protée
}

\section{Présentation : Échos et résonances}

\section{Catherine Mavrikakis et Catherine Morency}

Volume 35, numéro 1, printemps 2007

Échos et résonances

URI : https://id.erudit.org/iderudit/015883ar

DOI : https://doi.org/10.7202/015883ar

Aller au sommaire du numéro

Éditeur(s)

Département des arts et lettres - Université du Québec à Chicoutimi

ISSN

0300-3523 (imprimé)

1708-2307 (numérique)

Découvrir la revue

Citer ce document

Mavrikakis, C. \& Morency, C. (2007). Présentation : Échos et résonances. Protée, 35(1), 5-10. https://doi.org/10.7202/015883ar d'utilisation que vous pouvez consulter en ligne.

https://apropos.erudit.org/fr/usagers/politique-dutilisation/ 
PRÉSEN TATIO N

\title{
ÉCHOS ET RÉSO N ANCES
}

\author{
CATHERINE MAVRIKAKIS et CATHERINE MORENCY
}

\section{ÉCHOS DU CONTEMPORAIN}

Le fait d'être deux femmes ici possédant le même prénom, les mêmes initiales, n'est peut-être pas étranger à notre désir de penser à l'écho à divers moments dans les dernières années. Catherine Mavrikakis et Catherine Morency ont donc voulu réfléchir à la question de l'écho sans se répéter mais pourtant en écho. Il s'agit de tenter de saisir ce qui s'y dit ou s'y dédit, aussi bien que ce qu'il permet de dire - de la sériation (post)moderne (ou contre elle), de la création comme espace de résonance des rêves ou pensées d'un autre, et, surtout, de l'ouverture constitutive du geste littéraire contemporain aux autres textes, aux autres médias. Dans L'Entretien infini, Blanchot citant les mots de Nietzsche ouvre son livre ainsi: "Mais pourquoi deux? pourquoi deux paroles pour dire une même chose?" C'est que celui qui l'a dit c'est toujours l'autre» (1969: v). C'est toujours dans l'écho que quelque chose du sujet parle, dans le décalage d'une voix qui se fait entendre à nouveau, autrement.

\section{NÉCESSITÉS (POST)MODERNES DE L'ÉCHO}

Est-il possible de penser la modernité sans évoquer ce que Walter Benjamin a théorisé comme la perte de l'aura, sans la possible disparition d'une unicité, sans l'effacement d'une singularité d'un être, d'un objet ou encore d'un corps? À cette époque de reproductibilité technique, de reproductions assistées par les technologies de toutes sortes, de « réplication » de la vie même, est-il encore permis de croire à la saisie d'une originalité, d'une signature? Et n'est-ce pas dans la multiplication, la prolifération, la reprise que désormais nous sommes condamnés à penser le singulier ou ce qu'il en reste dans les chaînes de montage que sont devenues les existences?

Si les réseaux de fast-food à travers le monde clonent indéfiniment le même restaurant qui ne renvoie à aucun original réel, si, de Singapour à Brazzaville, les hôtels donnent partout naissance aux mêmes chambres aux lits à couvertures et moquettes synthétiques, si les guerres se multiplient en se répétant, en se voyant être deux fois mondiales en moins de trente ans et deux fois en Irak en moins de quinze ans, si les présidents américains se doublent de père en fils pour refaire le même scénario et arborent le même nom à une lettre près qui les distingue à peine et qui porte d'ailleurs en elle le redoublement, si les films se font sur le principe de la reprise (Star Trek XI sortira en 2008, nous annonce-t-on déjà), force est de constater que ce qui caractérise le temps actuel, c'est un désir de production du même, du sériel qui relève d’une véritable pauvreté de la pensée fondée sur un sentiment de peur devant ce qui est inconnu ou qui n'a pas déjà eu lieu. Le futur sera la copie de notre présent, voilà ce que les bandes-annonces des films prédisent. Le sentiment de « déjà-vu » des modernes, dont parle le philosophe Paolo Virno, s'explique non seulement par le cynisme d'un sujet blasé, mais aussi par l'impossibilité, qui est le lot actuel du social planétaire, de se donner de nouvelles formes de pensée, d'esthétique ou d'espoir. L'histoire ne peut que se répéter pour qu'elle puisse croire qu'elle a le mot de sa propre fin.

Face à cette folie du même, qui influence jusqu'à notre compréhension du temps historique, face à cette volonté d'entrer dans le clonage du présent que l'on conserve pour mieux le vivre plus tard, il semble important de 
penser les lieux de résistance au narcissisme forcené du redoublement, de la reprise. Il s'agit non pas de refuser l'inévitable et peut-être nécessaire marche vers la démultiplication, mais de parvenir à penser la question du double, de la doublure, de la répétition en tant qu'inquiétante étrangeté et non en tant que repère, antre pour un moi hypertrophié. La copie et la reproduction doivent rester le produit d'une altération, d'une trahison et d'une interprétation qui permet l'inscription d'une altérité imprévue.

Nous avons choisi l'écho pour en faire un concept permettant de penser le double dans l'idée d'une distorsion. Il nous a semblé, en effet, que c'était en lui que nous pouvions opposer un refus à l'idée du "cloné». Le psychanalyste, qui vient ponctuer certaines phrases de ses patients par des respirations, des expectorations, des soupirs, des raclements de gorge ou encore par une répétition écholalique des mots qui lui sont dits, parvient, par sa capacité à faire de son cabinet une chambre d'écho, à faire écouter au sujet quelque chose de sa propre parole jusque-là répétée comme même et donc non entendue. Grâce à ce modèle ou à cette fable psychanalytique où le «narcisse » du patient est tué par l'écho du psychanalyse, nous avons décidé de faire confiance à ce concept et de demander à d'autres de lui faire écho, de le penser, le répéter tout en se l'appropriant, le détruisant aussi partiellement.

L'écho, possible reprise déformante, renvoie le sujet à sa propre étrangeté et, ainsi, nous semble porter en lui l'éventuelle destruction d'un narcissisme tentaculaire et fascisant. Il peut donc être aussi perçu en tant que bruit, produit d'un parasitage, interférence, dysfonctionnement qui, au cœur même de la répétition, vient détraquer celle-ci.

C'est sur le mode de la maladie, du dérèglement qu'il faut alors penser l'écho, tel un virus qui vient infecter la logique du sériel tout en y participant. L'écho, en tant que reprise, opère une dramatisation, une «hystérisation» de la parole originale qui se met à dérailler, à être en excès, ou en manque, à produire son incohérence. Il substitue à l'idée de double une théâtralisation de la parole ou du sujet qui permet de quitter la «sérigénie » souvent mortifère. Ce concept nous conduit peut-être à penser de nouveau à l'être et à son aura. En effet, l'écho est ce qui prolonge la voix en montrant de celle-ci l'évanescence, la fragilité, la possible disparition. L'écho serait lié à la mort, à ce qui revient au sujet dans un travail d'effritement de sa propre présence. Il donnerait à entendre non pas la permanence de soi dans l'absence (ainsi qu'on le voit désormais dans la photo et peut-être à tort), mais bien plutôt la précarité de l'être-au-monde, qui fait de la voix un double évanescent. C'est le travail de la mort, de la possible décomposition du sens qui est inscrit dans l'écho, alors que toute copie nous semble être pensée actuellement sur le mode d’un refoulement de la vulnérabilité du sujet. La copie barricaderait le moi en le dédoublant.

Or, si l'écho semble être une figure permettant de briser l'édification narcissique qui, telle une mitraillette, fonctionne à répétition, il apparaît tout aussi important de réfléchir sur le statut accordé à la dissonance, à la disharmonie dans la modernité. Bien sûr, il pourrait être douteux et même odieux de se méfier d'un sujet altéré, métissé, mais il appert qu'il est nécessaire de s'interroger sur cet éloge collectif et surtout produit par les élites, de la béance du sujet et de sa résonance. On se fait ici l'écho, sûrement très infidèle, des travaux de Simon Harel qui, depuis des années, incitent à penser et repenser ce concert, pour le coup harmonique, d'éloges de la dissonance et de la diversité, au cœur de nos discours sociaux et de nos dispositifs de savoirs. La critique littéraire moderne, particulièrement au Québec, s'est fondée sur cette apologie du disparate, de l'interférence, de la discordance et du métissage. Cela ne nous a pas empêchés d'adopter une écoute pour le moins autiste des œuvres. Il ne saurait donc y avoir une naïveté fondamentale face au concept d'écho. Au contraire, si l'écho est un concept qui nous permet de briser la reproduction du même, il ne doit, en aucun cas, être le mot d'ordre d'une nouvelle foi et nous empêcher de penser ce que nous faisons lorsque nous convoquons sa capacité de résistance au monde moderne. 


\section{HOSPITALITÉS DE LA VOIX CRÉATRICE}

Dans le livre troisième d'Aurore, Nietzsche avance que les entreprises audacieuses sont plus rares dans les temps modernes que dans l'Antiquité et le Moyen Âge - probablement, propose-t-il, parce que les temps modernes ne croient plus aux présages, aux oracles, aux astres et aux devins. Selon lui, cela signifierait que nous sommes devenus incapables de croire qu'un certain avenir nous soit destiné, comme le faisaient les Anciens qui - à l'inverse de nous - étaient beaucoup moins sceptiques à l'égard de ce qui devait advenir que de ce qui existait.

Depuis 1867 , la parole est constamment menacée par ce scepticisme exacerbé, alimenté à l'aune d'un complexe devenu généralisé à l'endroit du lyrisme, jugé suspect par une âme détruite d'avoir vu sa pensée se penser et n'ayant plus la force que «d'évoquer en un Néant unique le vide disséminé en sa porosité » ${ }^{1}$. Si Mallarmé partage avec ses contemporains la frayeur de se voir émerger dans l'aura baudelairien sans parvenir à le transcender, c'est peut-être du côté d'une solitude obsessive qu'il faut chercher les causes de la crise asséchante, la naissance d'une épidémie qui se répandra ensuite partout en Occident et que les surréalistes tenteront tant bien que mal d'éradiquer en en dynamitant la racine: Narcisse court-circuité par un désir plus grand que le principe même qui avait généré, chez Gœethe, le fantasme de Werther - et tout poète supposément digne de ce nom après lui -, soit ce mirage ridicule de celui qui croit que création et individualité pourraient se féconder sans se contaminer, dans un concert productif doublé de son nécessaire avortement, puisque advenir seul (ou, plus prosaïquement, par soimême) constitue un syllogisme aussi bancal que mourir dans autrui.

Certes, Narcisse sera fatalement puni d'avoir trop contemplé sa superbe, mais d'abord et avant tout de s'être montré indifférent à la passion qu'il suscitait chez les autres, passion qui aurait pu devenir le sein d'un échange fertile plutôt que du dialogue stérile qui fut, tour à tour, son ultime chance de rédemption et son talon d'Achille. Dans le geste d'Écho, dans son désir et dans son élan, il y a bien un don, un gage maladroit et puéril de l'amoureuse qui veut sauver le garçon de l'écueil égotiste, l'étreindre de sa voix faute de mieux, et c'est cette abnégation, la nature irréductible de l'offrande, qui perturbera souvent, tel le pouvoir mystérieux des astres ou l'action du devin, le lecteur contemporain, dont le scepticisme évolue souvent jusqu'au mépris.

S'il boit du bout des lèvres, alter ego mal dégrossi de Paul Demeny, « La Lettre du voyant» et qu'il admet, s'inclinant, mystifié devant l'ascendant rimbaldien, que JE est un autre, le lecteur contemporain fera rarement l'effort d'en décrypter la corolle: «Si le cuivre s'éveille clairon, il n'y a rien de sa faute. [...] j'assiste à l'éclosion de ma pensée: je la regarde, je l'écoute: je lance un coup d'archet: la symphonie fait son remuement dans les profondeurs, ou vient d'un bond sur la scène ${ }^{2}$. Voir, c'est donc aussi entendre, bondir et saisir l'appel qui pourra s'avérer armes miraculeuses, en autant qu'on sache encore capter la parole du poète et l'apprivoiser comme sienne, suivant le spasme d'une chorégraphie sonore où l'on reconnaîtrait à la muse un rôle autre que passif. Ainsi que les sorts lancés par Artaud à ses proches, ce souffle inquiétant parce qu'étranger deviendra peut-être familier à I'oreille de celui qui, à l'instar de Nietzsche, recommencera à croire au pouvoir des oracles. Car jeter un sort appartient tout de même, au-delà de l'aspect maléfique du geste intempestif, à la gamme du don, tentative de rejoindre l'autre en faisant de sa propre voix un projectile agissant sur une conscience en mouvement à laquelle on tente de restituer - ou de mettre en lumière - son unicité.

Ted Hugues aurait-il connu telle fortune littéraire s'il n'avait pas «squatté» les blancs laissés, dans son linceul, par la mère de ses enfants, après sa disparition? Paul Celan aurait-il écrit un seul de ses vers s'il ne s'était pas systématiquement abîmé dans l'impératif essentiel qui, fidèle à la langue pourtant chargée de la plus haute trahison que lui avait apprise sa mère, consistait à fournir à cette dernière - et, à travers elle, à tous les morts de I'Holocauste - une sépulture scripturaire, faute de mieux? Hervé Guibert eut-il franchi le cap de l'image sans le 
côtoiement quotidien des êtres qui, tour à tour aimés et détestés, le hantèrent tout en lui permettant incessamment, et jusque dans la mort, de se mettre au monde à travers le truchement malhabile et pourtant rédempteur de la littérature? Puis cet âge de la parole, ce chant nouveau traqué par le poète, comment serait-il venu nous charmer, si ce n'est en mangeant la tête du serpent, le dard et le venin avalés faisant jaillir de nouveaux mots pour nommer ces soleils sanglants entrevus par celui qui osa, un jour, se détourner de sa propre image, mu par une aspiration incertaine mais non moins démesurée parce qu'universelle, et en cela profondément archaïque?

C'est bien à la démesure et à l'archaïque que nous renvoie l'écho, au potentiel de séduction contenu dans la voix de cet autre qui, nous visitant, continuerait de faire de nous, tel que le consignait Artaud sur la dernière page de son dernier cahier (1948, n 406), cet envoûté éternel, rendu vulnérable par son ouverture, son hospitalité face à un inconnu qui, pour autant que nous lui laissons nous montrer la voie, pourrait nous fournir les clefs d'une plus lucide compréhension du monde.

\section{ÉCHOS LITTÉRAIRES AU CONTEMPORAIN}

L'art et la littérature se sont toujours fondés sur l'idée d'un écho plus ou moins perceptible ou manifeste. Dans le cas de l'imitation des Anciens, il s'agissait de fonder l'œuvre en tant que double et reprise d'un idéal donné par l'Histoire. Les Modernes qui ont prêché la rupture, la souveraineté et souvent l'autonomie esthétique n'ont pu échapper à des filiations imaginaires, des intertextualités avouées ou cachées, des allusions intertextuelles plus ou moins dites qui restent alors, pour un scientifique tel que Gérard Genette, souvent aléatoires et du domaine de

I'impression $^{3}$. L'écho deviendrait, dans cette perspective, un procédé d'une évidence indéniable, mais, en même temps, il serait ridicule et inefficace pour la critique puisqu'il reste trop flou. La critique, il est vrai, en s'écartant de l'orthodoxie de Genette, peut faire ses choux gras des échos et s'amuser à les repérer et à les répertorier afin d'échafauder des théories. Loin de nous l'idée de déplorer cette hérésie scientifique. En fait, il nous faut plutôt prendre très au sérieux Genette lorsqu'il résiste à la prolifération folle et incertaine de l'écho et à son peu de consistance pour la critique littéraire. Il nous permet de repérer ici un symptôme : sa peur devant la prolifération de l'écho dans la critique rend peut-être compte de la littérature moderne comme espace de résonance tous azimuts et de relations contaminantes des textes les uns par rapport aux autres. Il faudrait analyser cette idée de la multiplication des échos dans la littérature actuelle et voir, en elle, un processus affolé de légitimation de l'œuvre qui se fonde dans des échos fusant de partout et faisant entendre une cacophonie d'œuvres presque méconnaissables et altérées.

Les échos dans plusieurs textes très contemporains sont multiples, avoués et inavoués. La littérature actuelle s'inscrit ainsi non seulement dans l'Histoire, mais aussi dans son présent. Quelque chose a-t-il changé récemment dans la littérature? Depuis vingt ans, un éclatement des formes littéraires canoniques se montre davantage et conduit à une diversification des configurations de l'objet livre (recueils, revues, livres-photos, cédéroms, blogues, etc.) et à l'émergence de nombreux corpus plurigénériques et intermédiaux. En traquant l'intrusion de l'écho dans les œuvres écrites depuis vingt ans, nous serions tentées de nous demander si la littérature ne serait pas désormais condamnée à se définir dans un dialogisme qui fait appel non seulement à d'autres formes littéraires, mais surtout à d'autres disciplines artistiques ou encore à de nouveaux espaces sortant totalement de l'ère de la poésie pure évoquée par Claude Gauvreau. Puisque l'écho réfléchit un son par un obstacle qui le répercute, ce phénomène nous semble emblématique des voix, spectrales ou incarnées, qui tentent aujourd'hui d'émerger du littéraire en s'entrechoquant aux autres formes de langage.

L'écho, figure polymorphe, se déploie- implicitement ou explicitement-dans de nombreux corpus contemporains, qui ne sauraient s'ériger en dehors d'un dialogue avec soi et avec l'autre, mais aussi avec les composantes culturelles d'un livre en mutation. À l'instar de Jean-Michel Rey, on peut se demander si l'œuvre 
contemporaine n'est pas dans l'obligation de s'apercevoir des limites qui sont les siennes et d'en passer par l'autre, une altérité qui s'impose dans le processus même de l'écriture. Cet autre, dont parle Rey, serait ici à penser dans I'espace des formes artistiques. L'écho peut donc être envisagé tout aussi bien sur le mode du parasite que sur celui de l'institution, de la dette à payer qui permettrait une certaine légitimité. Il confère au texte un nouveau statut, en lui permettant de transcender le cadre de l'espace littéraire et de re-fonder un nouvel ordre culturel qui l'autorisera à se constituer à travers la confrontation ou le dialogue avec les autres formes artistiques.

On songe, par exemple, à un Guyotat qui, en sortant Progénitures, donne à entendre son texte lu par lui sur un cédérom, à Hervé Guibert et à son roman-photo Suzanne et Louise et à tant d'autres écrivains contemporains qui font du livre une chambre de résonance pour d'autres formes d'art. Le processus d'engendrement des textes, en détournant ou en alimentant la fonction évolutive de la littérature, semble être alors modifié et vient transformer l'esthétique du livre. Fortes de ces questions sur la littérature dans la modernité et plus particulièrement à l'heure actuelle, nous avons demandé aux participants à ce numéro de Protée de venir penser l'écho à travers des textes. Il s'agissait, paradoxalement, de défaire l'inévitable demande de répondre par du même à nos questions. Nous invitions donc des collègues, critiques, penseurs, philosophes à reprendre, en les déformant, les interrogations qui étaient les nôtres. Ainsi, les participants ont répondu de façons diverses et personnelles, tout en créant, entre eux, certains effets d'écho.

Alain Milon nous permet de penser l'écho dans un réseau de concepts par lesquels il est possible d'appréhender le monde, le sujet et le langage. Il insiste sur le processus d'altération que l'écho constitue. II s'agit de voir comment l'écho « ouvre les failles du sujet dès l'instant où il se met à parler ». Travaillant sur les figures de la bifurcation et de la traduction, Milon veut mettre au jour l'altérité qu'est pour le sujet sa propre parole et réveiller ainsi un «lointain intérieur», toujours plus lointain. Le métissage engendré par l'écho, métissage posé ici comme altération, voire explosion de soi, met en scène la fêlure de la voix au cœur de tout travail, classique ou moderne, sur la langue.

$\mathrm{Si}$, pour Simon Harel, l'écho suscite trouble et ravissement, la dépossession qui est en jeu met aussi en scène des pensées anxieuses. En cela, Harel adopte une position plus critique face au concept d'écho. La perte de la source d'un certain «fracas sonore» peut conduire à des émois complexes (c'est le cas du délire). À partir de La Plâtrière de Thomas Bernhard, Harel montre le combat que se livrent le silence et le bruit dans l'écriture, puisque I'acte de penser est à la fois méditatif, silencieux et parasite. Réfléchissant ainsi sur le métissage et la diversité, Harel pose la question du silence que l'on tient à garder sur la dissonance des voix dans la mondialisation. Bernhard, s'irritant contre la vie, cherche un lieu pour écrire afin de faire taire l'écho, le bruit-parasite qui provoque l'agacement et la migraine. L'œuvre en sa qualité de demeure architecturale tentera de tuer l'écho tout en le reproduisant.

C'est en écho, dans un entrelacement créateur de leurs voix que Martine Delvaux et Jamie Herd réfléchissent sur deux œuvres-sœurs: Sœurs, saintes et sybilles de Nan Goldin et Autoportrait en vert de Marie Ndiaye. À partir $d^{\prime}$ une analyse de «l'usage conjoint des mots et des images photos », Delvaux et Herd tentent de voir comment une pratique féminine de l'écho, qui fait entendre la voix de la femme et apparaître l'infigurable féminin, s'installe dans une structure de résonance, de répétition et de doublure, où la reproduction (du réel) est sans cesse remise en question. C'est l'impossible figuration que soulignent ici les auteures de cet article. En effet, elles s'interrogent sur la possibilité de mettre en image une voix, de faire voir ce qui est trop montré et qui, paradoxalement, n'arrive pas à devenir l'objet d'un véritable regard: le féminin.

Thierry Bissonnette cherche, à travers les œuvres et la vie de Claude Gauvreau, à cerner quelques figures de l'écho qui conduisent l'auteur à mieux construire son identité esthétique. Gauvreau nous permettrait de penser 
l'écho comme emprise d'un spectral mais surtout en tant que travail sur la forme, puisque c'est l'écho qui décide des mécanismes et des structures de l'œuvre. Le père militaire apparaît ainsi dans la poésie du fils, «mitraille phonématique » productrice d'échos. La répétition permettra, dans ses infidélités, de donner lieu à une résurrection des morts, tout en légitimant une forme artistique d'avant-garde.

L'écho prend un tout autre sens dans un contexte où la voix, la résonance et le dédoublement sont maîtrisés par l'écrivain et font partie d'un système de contrôle de l'œuvre et de sa réception. Marie-Pier Luneau montre ici les stratégies, postures et impostures de Romain Gary/Émile Ajar pour renaître de ses cendres et se redonner vie au sein de l'institution littéraire et face à son public. Gary aurait mis lui-même au point un dispositif littéraire qui a amené les critiques de l'époque «à une lecture conservatrice des textes publiés sous son nom ». L'écrivain aurait ainsi contrôlé les échos publics de son travail.

C'est précisément sur la question du destinataire ou encore du récepteur de l'écho que Marie-Pascale Huglo travaille en poursuivant deux analyses: celle d'une fable d'Ovide où I'on voit évoluer Narcisse et Écho, et de celle de Saint Glinglin de Raymond Queneau. Elle montre comment l'écho implique nécessairement une réception. Ce qui intéresse ici Huglo, c'est la compréhension du stratagème formel de la répétition permettant « de faire entendre ce que l'on n'attendait pas». Dans la banalité des conversations représentées par Queneau dans son roman, l'écho fait voir le rituel de la répétition et perturbe «le mimétisme présumé » de la scène en hissant le dialogue au niveau de la poésie. Ainsi la parole est « rendue à la puissance élémentaire de la prolifération».

Faisant advenir ce qui ne pouvait avoir lieu, l'écho nous renverrait sans cesse à la question de l'origine. En pensant l'écho dans la perspective d'une « ontologique perception», Sylvie Boyer s'attache à voir en celui-ci les liens qu'il entretient avec la naissance. «Michel Leiris fut un enfant de remplacement ». C'est par cette affirmation, celle de l'enfant dédoublé, que débute le texte de Sylvie Boyer où il est question de réfléchir sur la présence $d^{\prime}$ un impensé intrafœetal dans l'œuvre de Leiris. Boyer cherche à entendre, dans l'œuvre de l'écrivain, l'appel des pleurs de la mère, de la "plainte funèbre», à la fois enchanteresse et effrayante - «écho de l'autre et de soi ». Elle voit

aussi la résistance de l'écrivain à cette voix maternelle qui « appelle l'enfant de remplacement comme autre». Le lieu prénatal sera alors l'espace où «s'amorce la faculté d'écoute de l'autre et de soi ».

\section{NOTES}

1. Extrait d'une lettre de Mallarmé adressée à Villiers de I'Isle-Adam le 24 septembre 1867 (Mallarmé, 1995: 366).

2. Lettre du 15 mai 1871 à Paul Demeny (Rimbaud, 2001: 250).

3. Le texte de Marie-Pascale Huglo présenté ici développe beaucoup mieux que nous ne saurions le faire cet argument.

\section{RÉFÉREN CES BIBLIO G RAPH IQ U ES}

BENJAMIN, W. [(1939) 2003] : L'OEuvre d'art à l'époque de sa reproductibilité technique, Paris, Éd. Allia. BLANCHOT, M. [1969] : L'Entretien infini, Paris, Gallimard.

GenetTe, G. [1982]: Palimpsestes. La littérature au second degré, Paris, Seuil, coll. «Points essais ».

GUIBERT, H. [1980] : Suzanne et Louise, Paris, Gallimard.

GuYOtAT, P. [2000] : Progénitures, Paris, Gallimard.

Mallarmé, S. [1995] : Correspondance. Lettres sur la poésie (dir. de B. Marchal, préf. d’Y. Bonnefoy), Paris, Gallimard, coll. «Folio classique».

NIETZSCHE, F. [1989] : Aurore, Paris, Gallimard, coll. «Folio essais».

ReY, J.-M. [2003] : Les Promesses de l'œuvre, Paris, Desclée de Brouwer.

RimBAud, A. [(1972) 2001] : OEuvres complètes, Paris, Gallimard, coll. "Bibliothèque de la Pléiade».

VIRNO, P. [1996]: Miracle, virtuosité et déjà vu. Trois essais sur l'idée de «monde», trad. de M. Valensi, Paris, Éd. de l'éclat. 